\title{
UN SPECTROMETRE ALPHA DESTINE A LA MESURE DE L'EQUILIBRE RADIOACTIF ENTRE LE RADON ET SES DESCENDANTS DANS UNE MINE D'URANIUM
}

\author{
Alain CHAPUIS, Louis LANEGRASSE et Alain LOPEZ * \\ (Manuscrit rę̧u le 16 juin 1970 )
}

\begin{abstract}
SOMMAIRE
On présente la réalisation d'un spectromètre $\alpha$ autonome et portatif destiné à la mesure de l'équilibre radioactif entre le radon et ses descendants dans les mines d'uranium. Le détecteur est une jonction de silicium à barrière de surface de $0,5 \mathrm{~cm}^{2}$. Le système de comptage :

- détecteur,

- préamplificateur et amplificateur,

- circuit de comptage,

- alimentation

a été conçu de façon à contenir dans un module de $40 \times 38 \times 20 \mathrm{~cm}$.
\end{abstract}

\section{ABSTRACT}

The realization of an autonomous and portable $\alpha$ spectrometer for the measurement of the degree of radioactive equilibrium between $\mathrm{Rn-222}$ and its daughter products in the uranium mines is presented. The detector is a $0,50 \mathrm{~cm}^{2}$ silicon surface barriere detector. The entire counting system :

- detector,

- preamplificator and amplificator,

- scaler,

- power supply

has been designed to fit into a module of $40 \times 38 \times 20 \mathrm{~cm}$.

\section{INTRODUCTION}

Cet article complète celui qui vient d'être présenté dans “ RADIOPROTECTION » par MM. A. Chapuis, A. Lopez, C. Cabrol, J. Pradel et F. Billard sous le titre :

«Sur les caractéristiques des aérosols radioactifs présents dans les mines françaises d'uranium ».

* Laboratoires Associés de Physique des Aérosols - Centre de Physique Atomique et Nucléaire de l'Université, II8, Route de Narbonne, 31 - Toulouse. 
Rappelons que, dans les mines d'uranium françaises, le déséquilibre entre le radon et ses descendants a été, jusqu'à présent, mesuré par étude de la décroissance de l'activité $\alpha$ totale recueillie sur un filtre [r] [2]. Le RaA et le RaC' collectés sur un filtre émettent des particules $\alpha$ de 6 et 7,68 MeV qui sont détectées au moyen d'un scintillateur de $\mathrm{ZnS}(\mathrm{Ag})$. Cette différence d'énergie introduit des rendements de détection qui peuvent varier avec le seuil de discrimination de l'électronique associée.

Afin d'éliminer cet inconvénient, nous avons développé [3] une méthode utilisée par Fusamura [4] au Japon, Mc Curdy [5], Lindeken [6] aux Etats-Unis, LASSEUR [7] en France. Elle consiste à analyser par spectrométrie l'activité $\alpha$ de la membrane.

Des calculs de MARTz [8], repris par СНавот [9], ont montré que la précision obtenue sur les fractions d'équilibre au moyen de la méthode spectrométrique était supérieure à celle obtenue par la méthode du comptage $\alpha$ total.

L'utilisation de la méthode spectrométrique dans les mines d'uranium demande l'élaboration d'un matériel autonome, robuste, portatif, d'un fonctionnement simple permettant à des personnes non spécialisées d'effectuer des mesures systématiques en différents points de prélèvement.

Cet article présente la réalisation d'un spectromètre $\alpha$ à jonction $P N$ à barrière de surface, satisfaisant à ces caractéristiques. Cette jonction a été fabriquée, au laboratoire par le groupe que dirige M. Maurice PETEL.

\section{RÉALISATION DE L'APPAREIL}

Il s'agit d'un spectromètre autonome à 4 canaux, d'un emploi simple et facilement transportable (fig. $\mathrm{x}$ ). La figure 2 donne le schéma synoptique de l'ensemble.

IO LE SPECTROMÈTRE

\section{a) Le détecteur}

C'est une jonction de silicium à barrière de surface dont la surface utile est de $0,5 \mathrm{~cm}^{2}$. Pour une tension de polarisation de 90 volts, le détecteur présente une zone désertée profonde de I $50 \mu \mathrm{m}$, bien supérieure au parcours des particules $\alpha$ dans le silicium.

\section{b) Le préamplificateur (fig. 3)}

Il est constitué par un cascode d'entrée formé par les transistors $Q_{1}, Q_{2}$. Pour éviter une réaction de l'amplificateur sur la partie sensible du préamplificateur la sortie se fait à basse impédance à l'aide d'un circuit " Bootstrap » constitué par les transistors $Q_{2}, Q_{3}$ et une capacité de réinjection de ro $\mu \mathrm{F}$. La contre réaction est obtenue par un condensateur de $2 \mathrm{pF}$ situé entre l'émetteur du transistor de sortie $Q_{3}$ et la base du transistor d'entrée $Q_{1}$.

c) Amplificateur (fig. 4)

Le schéma de base est celui donné par EMmer [1o]. Il permet d'obtenir une impulsion positive d'amplitude égale à $200 \mathrm{mV}$ pour une énergie de $\mathrm{I} \mathrm{MeV}$. 
La figure s donne la hauteur d'impulsion en fonction de l'énergie des particules $\alpha$ émises par différentes sources placées à $7 \mathrm{~mm}$ du détecteur. La réponse de l'amplificateur est linéaire pour des énergies inférieures à I I $\mathrm{MeV}$.

d) Le sélecteur d'amplitude à 4 canaux (fig. 6)

Le sélecteur d'amplitude a été construit sur la base des études faites par Swift [ I I ] et Srmon [ I 2]. Il est constitué de quatre ensembles identiques, comprenant chacun un discriminateur et un circuit de sortie. Les impulsions à analyser sont envoyées simultanément sur les quatre circuits de discrimination constitués d'une diode tunnel TU $10 / 1$ et d'un transistor ASY 29 fonctionnant en monostable [13]. L'impulsion négative obtenue sur le collecteur de ce transistor a une amplitude de 6 volts et une largeur de is $\mu$ s que l'on règle à l'aide de la résistance de $3,3 \mathrm{k} \Omega$ et de la capacité de $\mathrm{I}, 2 \mathrm{nF}$. Ce signal négatif est différencié par la capacité de $200 \mathrm{pF}$ pour donner une impulsion positive sur la base du transistor de sortie. De plus ce signal négatif est intégré pour bloquer le canal inférieur. L'impulsion de sortie, obtenue sur le collecteur du transistor $2 \mathrm{~N} 708$ est une impulsion négative, large de I $\mu$ s environ, qui convient pour attaquer une échelle de comptage classique.

Les seuils de discrimination des différents canaux sont stables. Ils sont réglés au moyen des potentiomètres $K_{1}, K_{2}, K_{3}, K_{4}$. A chaque canal correspond une bande d'énergie bien déterminée (fig. 7). Le seuil supérieur d'un canal correspond au seuil inférieur du suivant. Sur le $4^{\mathrm{e}}$ canal enfin sont détectés tous les rayonnements $\alpha$ d'énergie supérieure à $E_{3}$.

\section{$2^{\circ}$ Circuit de comptage}

Les impulsions négatives obtenues à la sortie de chaque canal sont envoyées sur des monostables de mise en forme qui permettent de commander les numérateurs mécaniques et les décades à affichage numérique. Les circuits (Arrêt Marche - Remise à zéro) pour les décades électroniques sont de conception classique.

\section{$3^{0}$ Alimentation}

Les tensions d'alimentation sont obtenues à partir de trois accumulateurs (cadmium-nickel ( $12 \mathrm{~V}-\mathrm{I} \mathrm{Ah}$ ) et de deux accumulateurs (6 V - $4 \mathrm{Ah}$ ) régulées par des alimentations stabilisées. Un convertisseur donne la tension de 180 volts nécessaire à l'alimentation des tubes à affichage numérique et la tension de 90 volts nécessaire pour la polarisation du détecteur. Un chargeur à thyristors est incorporé à l'appareil.

Les accumulateurs utilisés donnent une autonomie de $5 \mathrm{~h}$ de marche en continu. On envisage d'apporter des améliorations afin d'augmenter la durée de fonctionnement.

\section{ÉTALONNAGE}

Les seuils des canaux sont réglés de façon que le premier mesure l'activité du $\mathrm{RaA}$ et le troisième celle du RaC'. Le seuil inférieur du premier canal est fixé par une source d'énergie faible (plutonium 239 d'énergie 5,1s $\mathrm{MeV}$ ) de façon 
à ne pas éliminer les particules $\alpha$ du RaA qui auraient perdu une partie de leur énergie dans le filtre de prélèvement ou dans leur trajet entre le filtre et le détecteur.

Les seuils des autres canaux sont déterminés en utilisant un filtre obtenu par aspiration sur une source de radium. Le seuil supérieur du premier canal est réglé de façon à ce que les impulsions dues au RaA ne soient pas comptées sur le deuxième canal. De même les seuils supérieurs des deuxième et troisième canaux sont réglés de façon à ce que les impulsions dues au $\mathrm{RaC}^{\prime}$ ne soient pas comptées sur les deuxième et quatrième canaux.

La figure 8 donne le spectre obtenu sur un sélecteur multicanaux d'un filtre ayant prélevé sur une source de radium. La distance filtre-détecteur est de $3 \mathrm{~mm}$. Dans ce cas la résolution est de $4,5 \%$ pour le RaA et de $3,5 \%$ pour le RaC'. Il faut noter l'influence du filtre et de la granulométrie des aérosols collectés sur la résolution. Des essais ont montré que la meilleure résolution était obtenue avec des filtres millipore type $\mathrm{PH}$, diamètre des pores $0,3 \mu \mathrm{m}$. Il est possible d'améliorer la résolution en faisant le vide dans l'ensemble de détection lors de la mesure.

\section{RENDEMENT DE DÉTECTION}

Le rendement de détection peut s'exprimer au moyen de la relation :

$$
\rho=\frac{d \Omega}{4 \pi} F_{t} \cdot F_{a} \cdot \rho d
$$

$\mathrm{d} \Omega$ étant l'angle solide sous lequel le détecteur voit la source,

$F_{t}$ un facteur de transmission,

$F_{a}$ un facteur d'auto-absorption,

pa le rendement du détecteur.

En première approximation le rendement de détection peut être pris égal au facteur de géométrie $d \Omega / 4 \pi$. En effet :

a) Le facteur d'auto-absorption dans la membrane peut être négligé. La perte d'énergie des particules $\alpha$ dans la membrane se traduit par un élargissement des pics vers les basses énergies. Le $\mathrm{RaC}^{\prime}$ est alors détecté sur les deuxième et premier canaux. L'élargissement du pic du RaA n'a aucune influence sur le rendement de détection, le seuil inférieur du premier canal est réglé à une énergie plus faible $(s, \mathrm{I} s \mathrm{MeV})$ que celle du RaA.

b) Le facteur de transmission peut être négligé. Les particules $\alpha$ du RaA et du $\mathrm{RaC}$, même partiellement absorbées, possèdent, dans l'air, un parcours supérieur à la distance du filtre au détecteur. L'absorption sur cette distance jouera donc un rôle sur la résolution, et non sur le rendement de détection.

c) Le rendement du détecteur est une fonction de l'énergie des particules $\alpha$. Pour une tension de polarisation de 90 volts il est pratiquement égal à l'unité pour les énergies du RaA et du RaC'.

La figure 9 donne la géométrie source-détecteur. La surface aspirante du filtre de prélèvement est de $9 \mathrm{~cm}^{2}$. Afin de bien définir la source, cette surface 
est limitée par un diaphragme de diamètre $2 \mathrm{~cm}$ lors du comptage. Avec les détecteurs utilisés, la distance filtre-détecteur était de $3 \mathrm{~mm}$.

Dans ces conditions, nous avons calculé, à l'aide d'une méthode de MonteCarlo le rendement théorique de détection (en fonction de la distance filtredétecteur). Les résultats sont donnés sur la figure ıo. Nous avons par ailleurs vérifié expérimentalement ces résultats.

Il est possible d'accroître la valeur du rendement de détection, donc la sensibilité et la précision des mesures, en employant des détecteurs de surface beaucoup plus grande (détecteurs mosaïques).

\section{CONCLUSION}

De nombreuses mesures ont été faites dans les mines d'uranium françaises au moyen du spectromètre $\alpha$ que nous venons de décrire $[3,9]$.

Cet appareil présente l'avantage d'être utilisable directement dans la mine, ce qui est nécessaire pour mesurer avec précision la concentration d'éléments à vie courte comme le radon.

Nous remercions MM. les Professeurs Blanc et Fontan ainsi que M. A. Birot du Centre de Physique Atomique et Nucléaire de Toulouse pour leur aide et leurs conseils.

Ces recherches ont été effectuées grâce à un contrat du Commissariat à l'Energie Atomique. Nous remercions MM. Pradel, Billard et Madelaine (Service Technique d'Etudes de Protection) pour leur collaboration et leur appui dans la réalisation de ce travail.

\section{BIBLIOGRAPHIE}

[i] Blanc D., Fontan J., Chapuis A., Billard F., Madelaine G., Pradel J. Assessment of airborne radioactivity, Rapport SM 95/3, IAEA, 229, Vienne (1967).

[2] Chapuis A., Lopez A., Fontan J. Colloque sur la physique des aérosols, Francfort (1969).

[3] Lopez A., Chapuis A., Fontan J., Billard F., Madelanne G. Aerosols science, Sous presse.

[4] Fusamura W., Kurosawa R., Maruyama M. Assessment of airborne rad., IAEA, 63i, Vienne (1967).

[s] Mc Curdy D. E. Rapport USAEC, n० C oo-1 soo, Fort-Collins (1967).

[6] LINDEKEN L. J. of Geopbys. Researcb, 73, no 8, (1968).

[7] Lasseur C. Thèse d'Université, $\mathrm{n}^{\circ} 253$, Toulouse (1970).

[8] Martz D. E., Holleman D. F., Mc Curdy D. E., Schinger K. J. Healtb Pbysics, 17, 131 (1969).

[9] Снавот A. Thèse de Doctorat de Spécialité, nº 899, Toulouse (1970).

[ro] Emmer T. L. I.R.E. Trans. Nucl. Sciences, 8, 140 (196r).

[i 1] Swift C. D. Rev. Sc. Instr., 34, 306 (1963).

[12] Srmon M. Diplôme, Université de Kiel (1967).

[13] Gorodetzky S., Muser A., Zen J., Armbruster R. Nuclear Instruments and Metbods, 13, 282 (196r). 

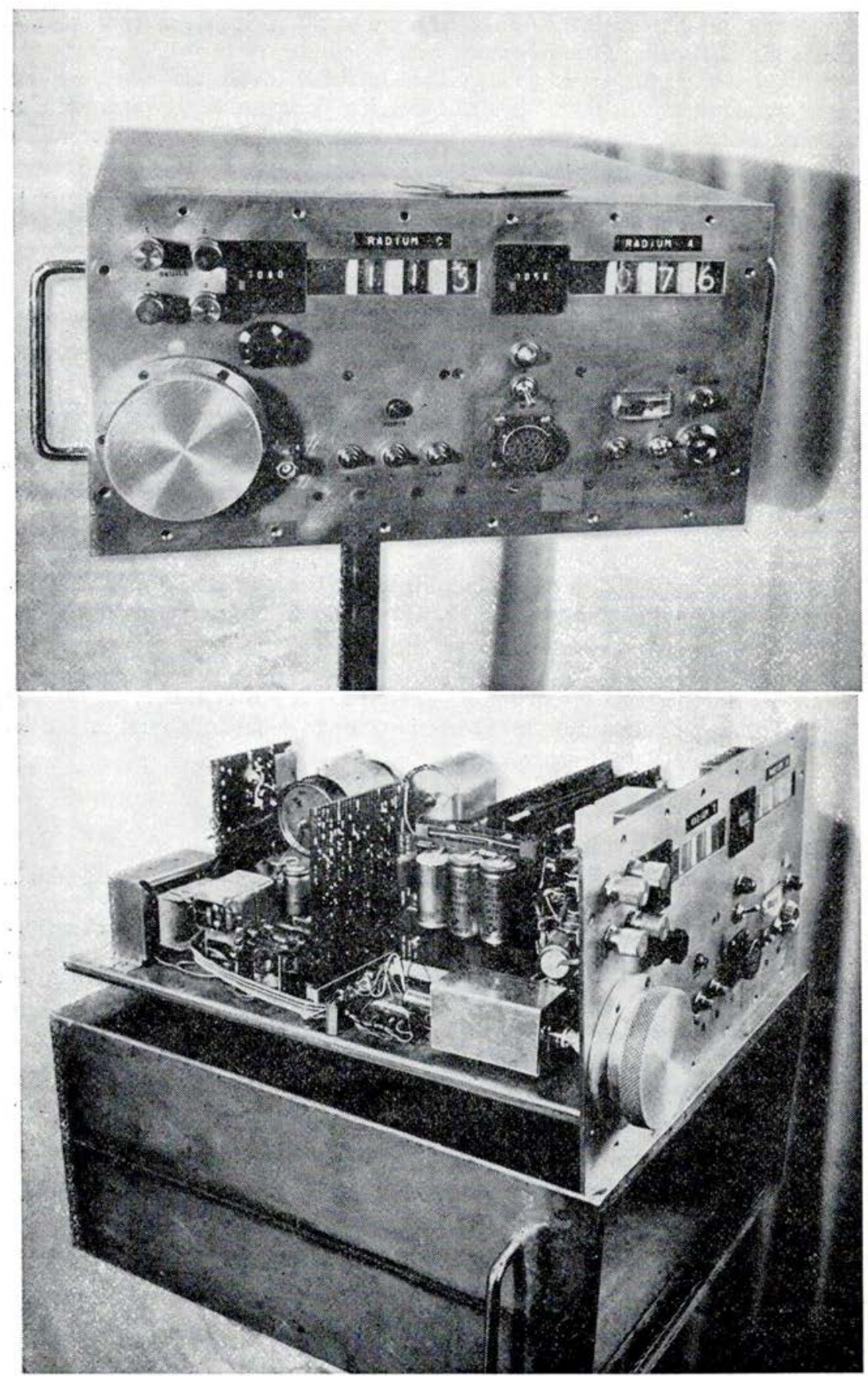

FIG. I. - Vue du spectromètre $\alpha$ (2 photos). 
( SPECTROMÈtre ALPHA POUR MESURE DU RADON DANS LES MINES ) 299

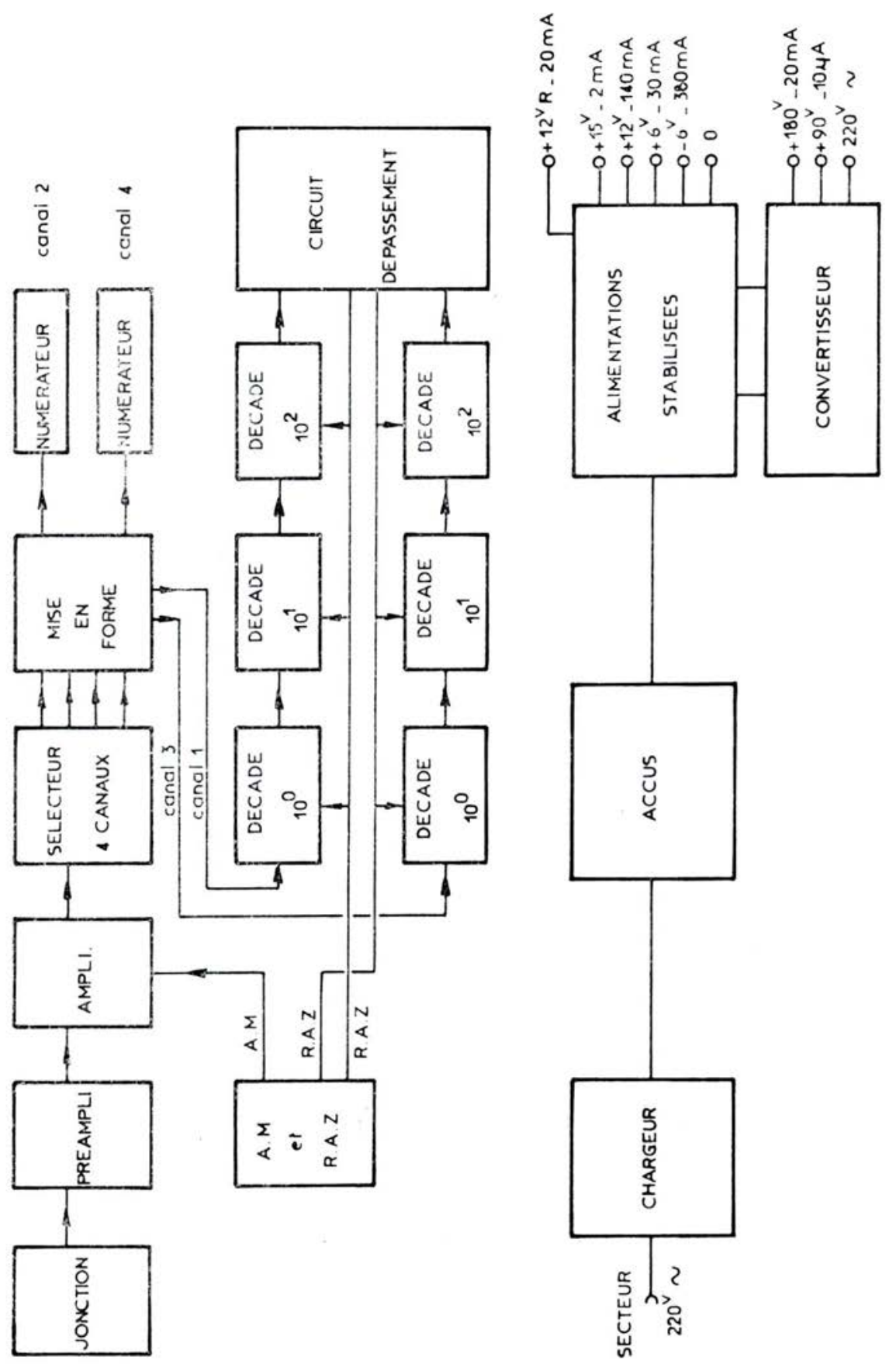

FIG. 2. - Schéma synoptique du spectromètre $\alpha$. 


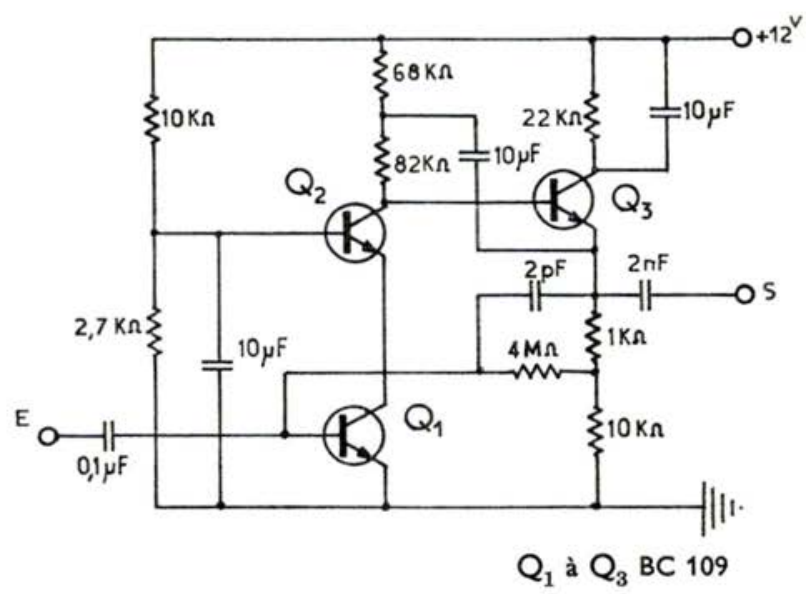

Fig. 3. - Schéma du préamplificateur de charge.

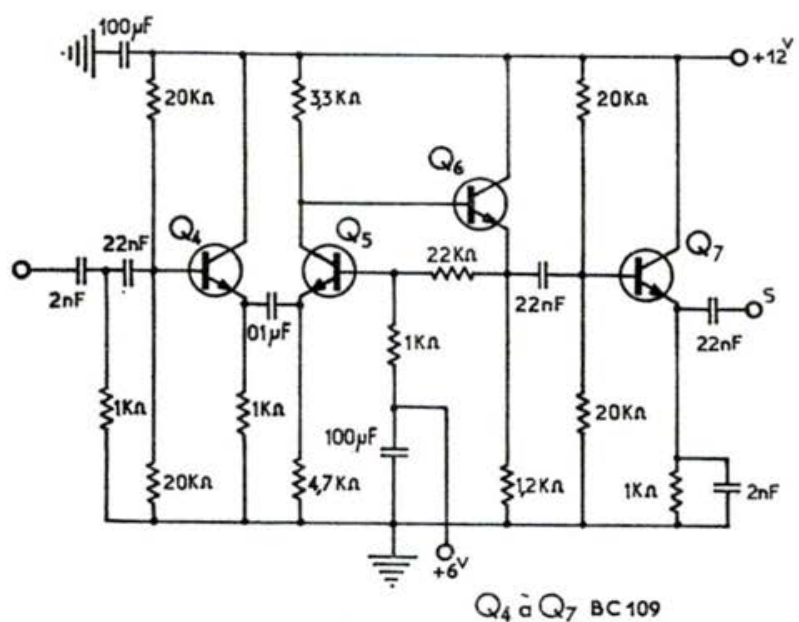

FIG. 4. - Schéma de l'amplificateur linéaire. 
( SPECTROMÈTRE ALPHA POUR MESURE DU RADON DANS LES MINES ) 30 I

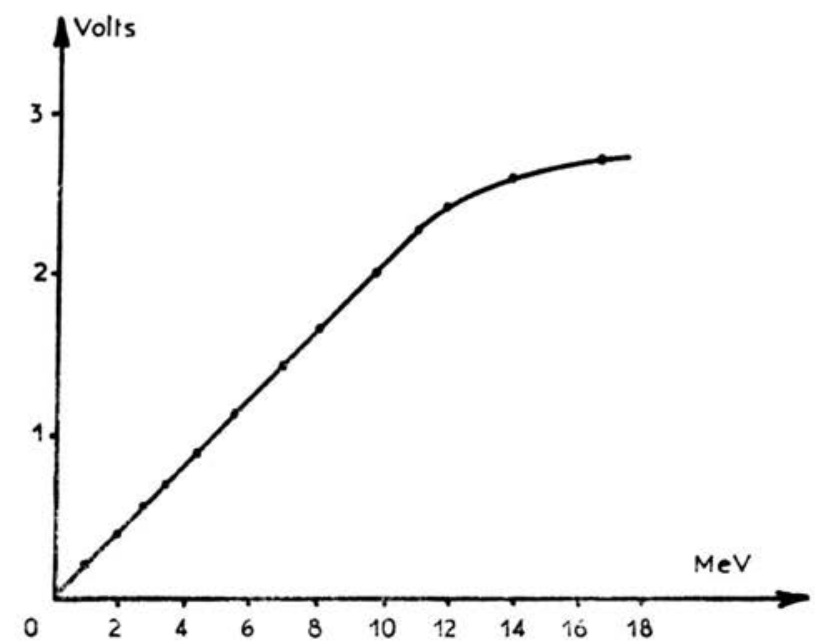

Fig. s. - Variation de la tension de sortie de l'amplificateur linéaire en fonction de l'énergie de la particule $\alpha$ incidente. 


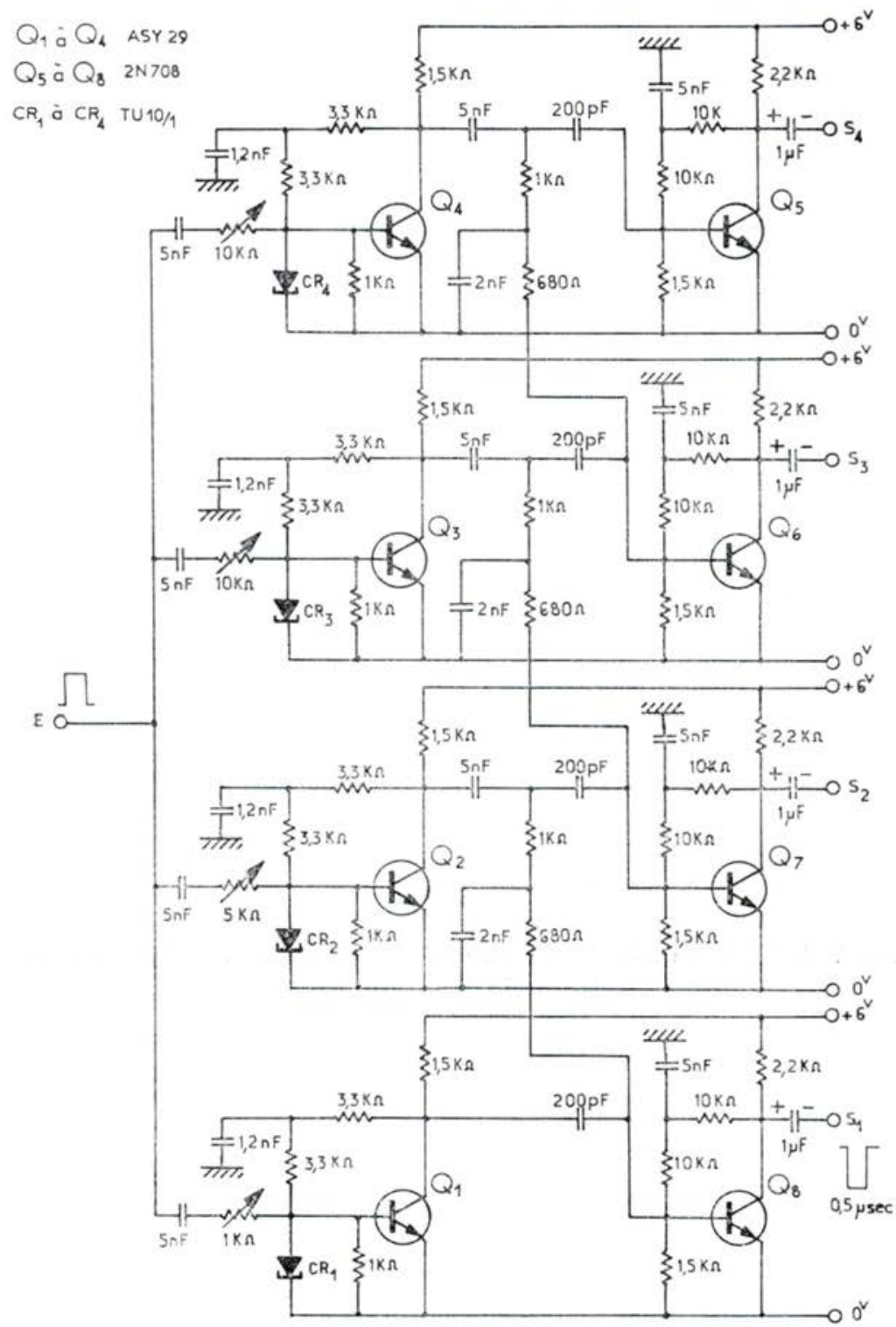

Fig. 6. - Schéma de l'analyseur d'amplitude à 4 canaux. 
« SPECTROMÈTRE ALPHA POUR MESURE DU RADON DANS LES MINES " 303

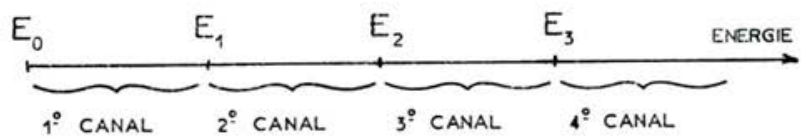

FIG. 7. - Situation des canaux en énergie.

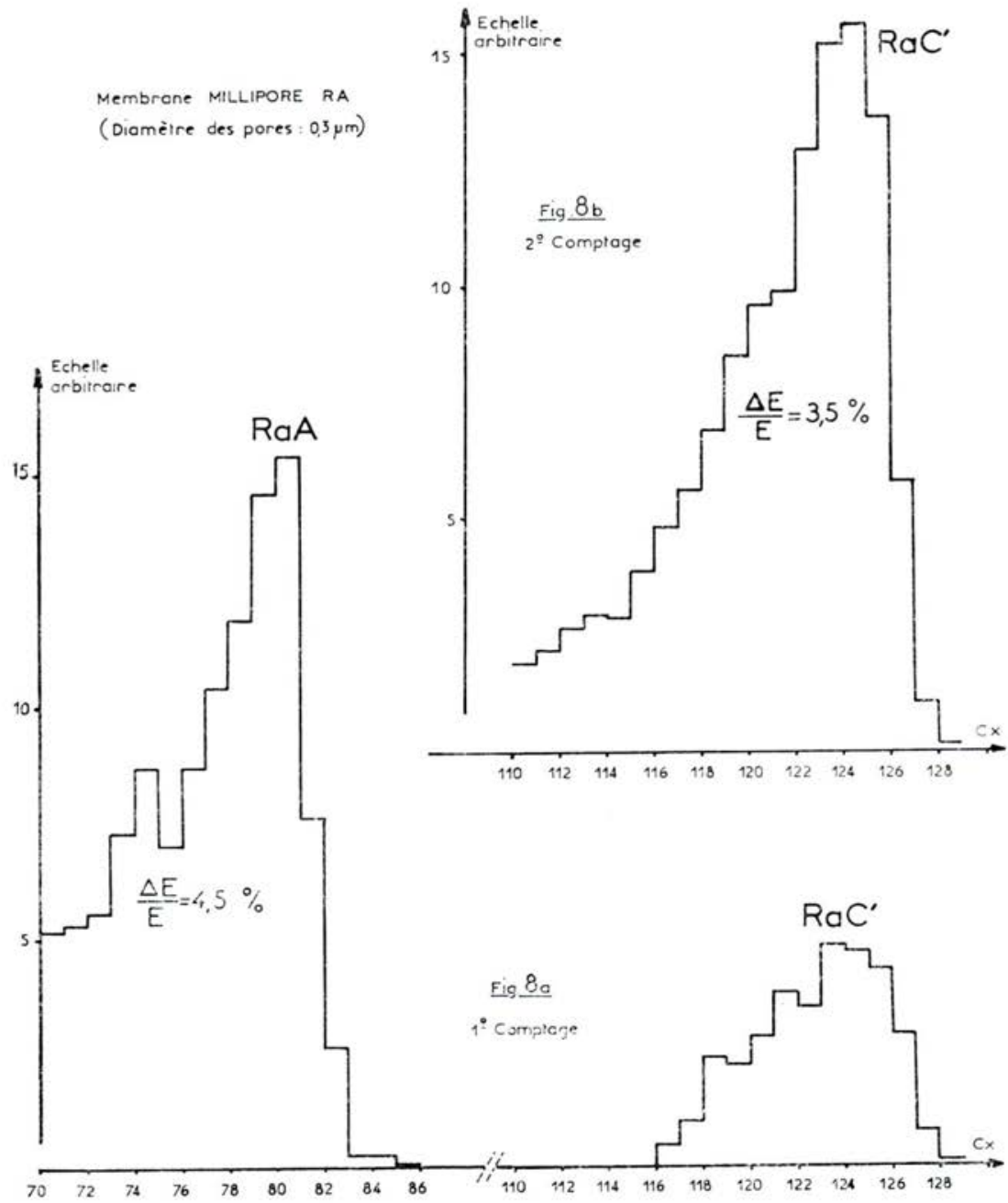

FIG. 8. - Spectre d'un filtre ayant collecté les descendants du radon

a) $\mathrm{I}^{\circ}$ comptage,

b) $2^{\circ}$ comptage. 


\section{PORTE FILTRE \\ AVEC \\ DIAPHRAGME}
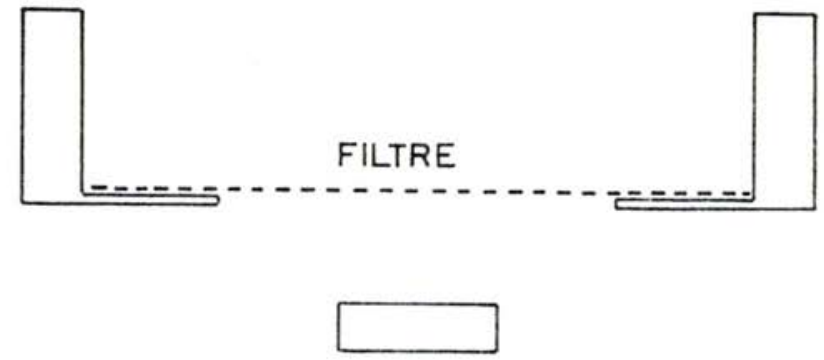

DETECTEUR

FIG. 9. - Géométrie de détection.

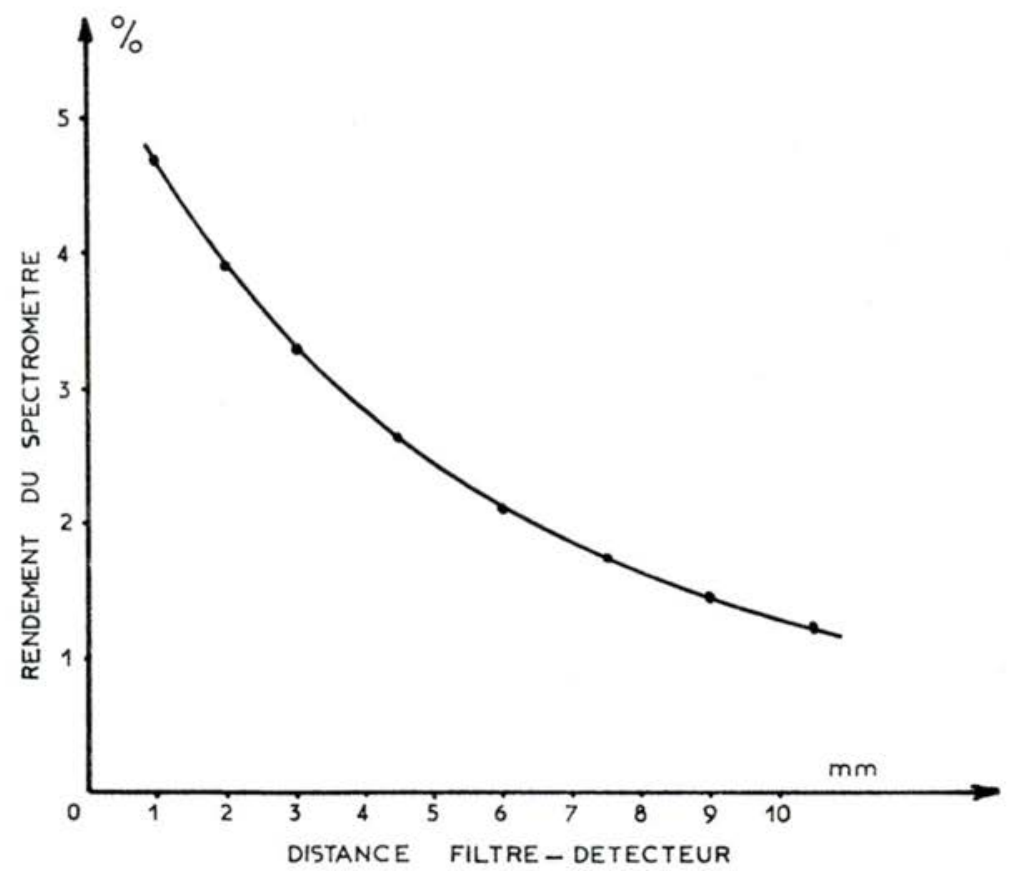

FIG. Io. - Variation du rendement théorique de détection en fonction de la distance filtredétecteur. 